\title{
Pediatric acute appendicitis: Ultrasound diagnosis
}

Greg Zahn and Julie L. Welch

Department of Emergency Medicine, Indiana University School of Medicine, Indianapolis, Indiana, USA

11 yo female presented to the ED for 2 days of nausea and abdominal pain that localized to the right lower quadrant. She had no fever, urinary symptoms, vomiting or diarrhea. Her vitals were a temperature of $99.5 \mathrm{~F}$ and heart rate of 118. Abdominal tenderness was maximal over McBurney's point, with voluntary guarding. Given a preference to avoid ionizing radiation, ultrasound was selected to evaluate for appendicitis. Our institution has limitations in obtaining formal appendix ultrasound studies; thus a bedside ultrasound was performed by a trained EM physician. At the point of maximal tenderness, a blind-ending, non-compressible structure was identified, with a maximal diameter of 1.2 $\mathrm{cm}$, consistent with acute appendicitis. (Figs. 1 and 2) A definitive diagnosis was obtained within minutes of the patient being in the ED. The child subsequently underwent an uncomplicated laparoscopic appendectomy confirming acute appendicitis. ${ }^{1-3}$

\section{Supplementary material}

Supplementary material associated with this article can be found, in the online version, at doi:10.1016/j.visj.2018.10.001.

This is the author's manuscript of the article published in final edited form as:

Zahn, G., \& Welch, J. (2019). Pediatric acute appendicitis: Ultrasound diagnosis. Visual Journal of Emergency Medicine, 14. https://doi.org/10.1016/j.visj.2018.10.001 


\section{References}

1. Fields M.J., Davis J., Alsup C., et al: Accuracy of point-of-care ultrasonography for diagnosing acute appendicitis: a systematic review and meta-analysis. Acad Emerg Med 2017; 24: pp. 1124

2. Mittal M.K., Dayan P.S., Macias C.G., et al: Performance of ultrasound in the diagnosis of appendicitis in children in a multicenter cohort. Acad Emerg Med 2013; 20: pp. 697

3. Goldin A.B., Khanna P., Thapa M., et al: Revised ultrasound criteria for appendicitis in children improve diagnostic accuracy. Pediatr Radiol 2011; 41: pp. 993 
Fig. 1. Clarification of video in Figure 1, shows outline of inflamed appendix.

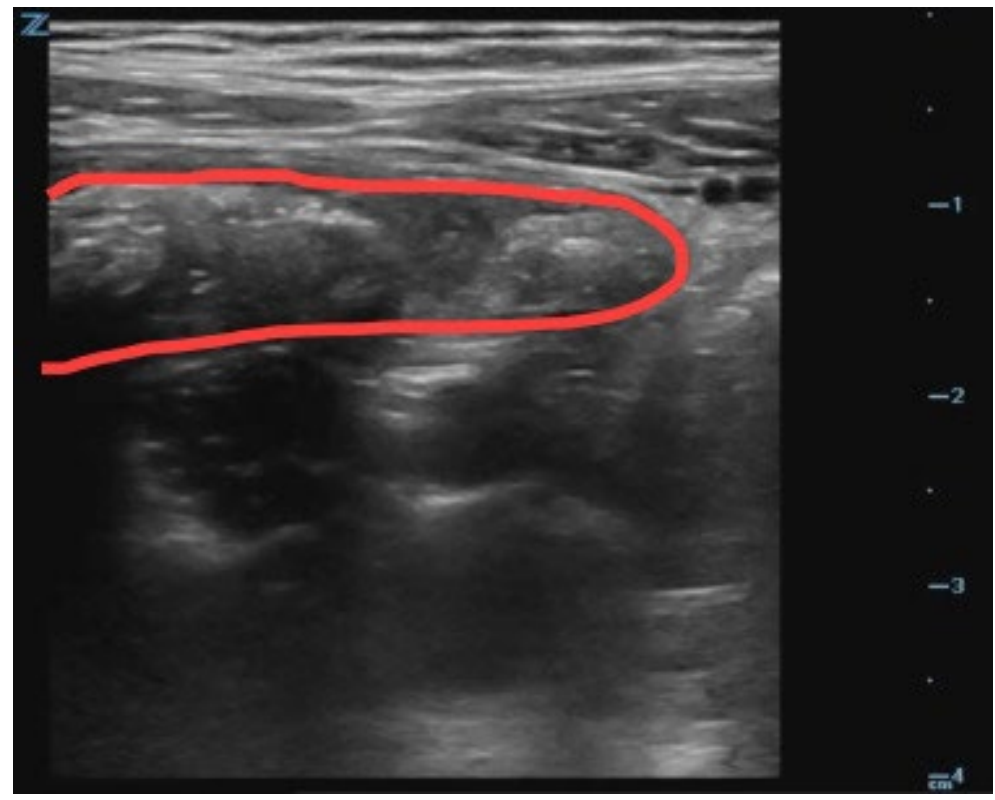

